\title{
Symbolverzeichnis Band III
}

\author{
(alphabetisch)
}

\begin{tabular}{|c|c|}
\hline$A_{H}$ & Hall-Konstante \\
\hline$\vec{A}(\vec{r})$ & Vektorpotenzial \\
\hline$a, \vec{a}$ & Beschleunigung $(a=\dot{v}=\ddot{r})$ \\
\hline$B, \vec{B}$ & magnetische Induktion (magnetische Flussdichte, „Magnetfeld“) \\
\hline$C$ & elektrische Kapazität \\
\hline$C_{C}$ & Curie-Konstante \\
\hline$C_{F}$ & elektrochemisches Äquivalent \\
\hline$C^{\prime}, L^{\prime}, R^{\prime}, G^{\prime}$ & Leitungsbeläge (Wellenausbreitung auf Leitungen) \\
\hline$c$ & Lichtgeschwindigkeit im Vakuum ( $c=299792458$ m/s) \\
\hline$D^{*}$ & Direktionsmoment \\
\hline$D, \vec{D}$ & Drehmoment \\
\hline$\vec{D}$ & dielektrische Verschiebung (elektrische Kraftflussdichte) \\
\hline$E, \vec{E}$ & elektrische Feldstärke \\
\hline$\vec{E}_{\text {Diel }}, \vec{E}_{\mathrm{Vak}}$ & elektrische Feldstärke im Dielektrikum bzw. im Vakuum \\
\hline$E_{\text {pot }}$ & potenzielle Energie \\
\hline$E K L$ & Klemmenspannung \\
\hline$E M K$ & elektromotorische Kraft (Quellenspanung) \\
\hline$e$ & Elementarladung \\
\hline$e^{-}$ & Elektron \\
\hline$F$ & Faraday Konstante $\left(F=N_{A} \cdot e=96485,3321233100184 \mathrm{C} / \mathrm{mol}\right.$, exakt) \\
\hline$F, \vec{F}$ & Kraft \\
\hline$\vec{F}_{C}, \vec{F}_{B}, \vec{F}_{E M}$ & Coulombkraft, Lorentz-Kraft, Lorentz-Gesamtkraft \\
\hline$H, \vec{H}$ & magnetische Feldstärke (magnetische Erregung) \\
\hline I & elektrische Stromstärke, Intensität \\
\hline$\vec{\imath}$ & „Zeiger“ für den Wechselstrom \\
\hline$I_{p}, I_{s}$ & Strom in der Primär-, Sekundärspule (Transformator) \\
\hline$I_{s}, \theta$ & Trägheitsmoment \\
\hline$I_{V}$ & Verschiebungsstrom $\left(I_{V}=\varepsilon_{0} \frac{d \Phi_{E}}{d t}\right)$ \\
\hline$j, \vec{j}$ & Stromdichte \\
\hline$k$ & Boltzmannkonstante \\
\hline$k, \vec{k}$ & Wellenzahl, Wellenvektor \\
\hline$L$ & Induktivität, Selbstinduktionskoeffizient \\
\hline$L, \vec{L}$ & Drehimpuls \\
\hline$L_{12}$ & Gegeninduktivität, Gegeninduktionskoeffizient \\
\hline$M, \vec{M}$ & Magnetisierung \\
\hline$m$ & Masse \\
\hline$m_{e}$ & Masse des Elektrons \\
\hline$m_{S}$ & magnetische Spinquantenzahl \\
\hline$N$ & Teilchenzahl \\
\hline$N_{A}$ & Avogadrozahl $\left(6,02214076 \cdot 10^{23} \mathrm{~mol}^{-1}\right.$, exakt $)$ \\
\hline$N_{\mathrm{p}}, N_{\mathrm{s}}$ & Windungzahlen der Primär- und Sekundärspule (Transformator) \\
\hline$n$ & Teilchendichte $\left(n=\frac{N}{v}\right)$ \\
\hline$P$ & elektrische Leistung $\left(P=\frac{a v V}{d t}\right)$, Polstärke \\
\hline
\end{tabular}




$$
\vec{P}
$$

$P_{S}$

$P_{\mathrm{W}}, P_{\mathrm{S}}, P_{\mathrm{B}}$

dielektrische Polarisaion

$p$

$p, \vec{p}$

$\tilde{p}$

$\vec{p}_{e}, \vec{p}_{m}$

$\vec{p}_{m}^{L}$

$\vec{p}_{m}^{S}$

$Q$

$\tilde{Q}_{2}$

$Q_{0}, Q_{1}, Q_{2}$

$q, Q$

$R$

$S, \vec{S}$

$S, \vec{S}$

$T$

$T_{c}$

$U$

$\vec{U}$

$U_{\text {ind }}$

V

$V_{\text {Mol }}$

$v, \vec{v}$

$v_{\mathrm{ph}}, v_{\mathrm{gr}}$

W

WW

w

$\vec{Z}$

$\vec{z}_{L}, \vec{z}_{C}$

$\left|z_{L}\right|,\left|z_{C}\right|$

$Z_{0}$

$\alpha$

$\beta$

$\gamma$

$\Delta$

$\vec{\nabla}$

Strahlungsdruck

Impuls

(beim Elektron $\mu_{e}^{L}$ )

(beim Elektron $\mu_{e}^{S}$ )

Quadrupoltensor

Ladung

Eigendrehimpuls (Spin)

induzierte Spannung

Volumen

Geschwindigkeit $(v=\dot{r})$

Arbeit, Energie

Wechselwirkung

Energiedichte $\left(w=\frac{w}{v}\right)$

Wellenwiderstand
Wirk-, Schein-, Blindleistung

Reflexionsfaktor (Wellenausbreitung auf Leitungen)

Impulsdichte (elektromagnetische Welle)

elektrisches und magnetisches Dipolmoment

durch den Bahndrehimpuls verursachtes magnetisches Dipolmoment

durch den Eigendrehimpuls (Spin) verursachtes magnetisches Dipolmoment

Gütefaktor (Schwingkreis)

Monopol-, Dipol-, Quadrupolanteil

elektrischer ohmscher Widerstand

Momentane Energiestromdichte, Poynting-Vektor

Umlaufzeit, Schwingungsperiode

Sprungtemperatur (Supraleiter)

elektrische Spannung (Potenzialdifferenz)

„Zeiger“ für die Wechselspannung

Molvolumen $\left(V_{\text {Mol }}=\frac{M}{\rho}, M\right.$... Molekulargewicht, $\rho$... Massendichte $)$

Phasen-, Gruppengeschwindigkeit

„Zeiger“ für den Wechselstromwiderstand

komplexer induktiver und kapazitiver Widerstand $\left(\vec{Z}_{L}=i \omega L, \vec{Z}_{C}=-\frac{i}{\omega C}\right)$

Impedanz einer Spule, Impedanz eines Kondensator $\left(\left|Z_{L}\right|=\omega L,\left|Z_{C}\right|=\frac{1}{\omega C}\right)$

Polarisierbarkeit, Temperaturkoeffizient (spezifischer elektrischer Widerstand)

Geschwindigkeitsparameter $(\beta=v / c)$

Gravitationskonstante, Lorentz-Faktor $\left(\gamma=\frac{1}{\sqrt{1-v^{2} / c^{2}}}=\frac{1}{\sqrt{1-\beta^{2}}}\right)$, Dämpfungs-

konstante (Schwingkreis), Fortpflanzungsfaktor (Wellenausbreitung auf Leitungen)

Laplace-Operator $\left(\Delta=\frac{\partial^{2}}{\partial x^{2}}+\frac{\partial^{2}}{\partial y^{2}}+\frac{\partial^{2}}{\partial z^{2}}\right)$

Nablaoperator $\left(\vec{\nabla}=\vec{e}_{x} \frac{\partial}{\partial x}+\vec{e}_{y} \frac{\partial}{\partial y}+\vec{e}_{z} \frac{\partial}{\partial z}\right)$

Permittivität (Dielektrizitätskonstante) 


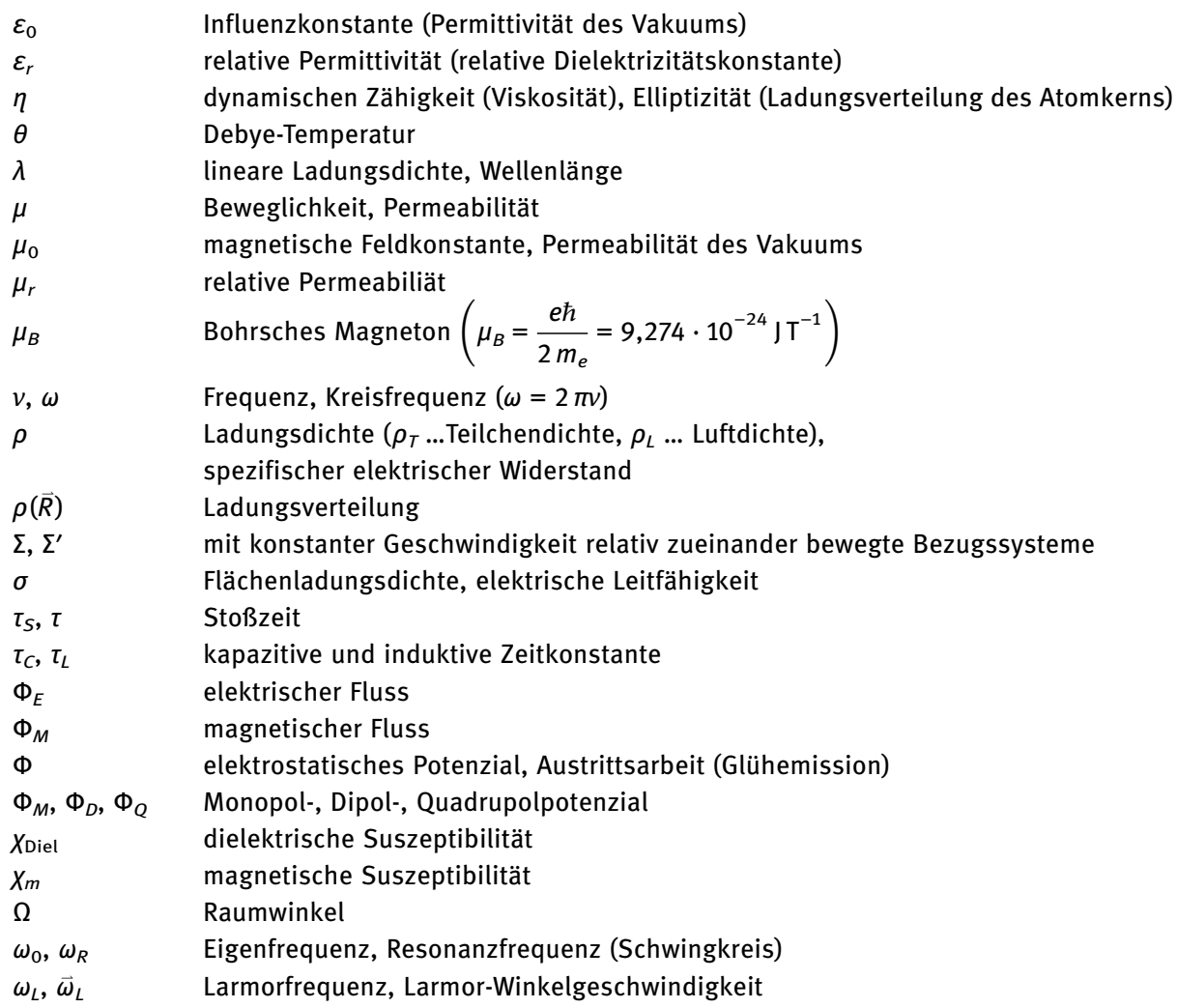

\section{Wichtige physikalische Größen, Band III}

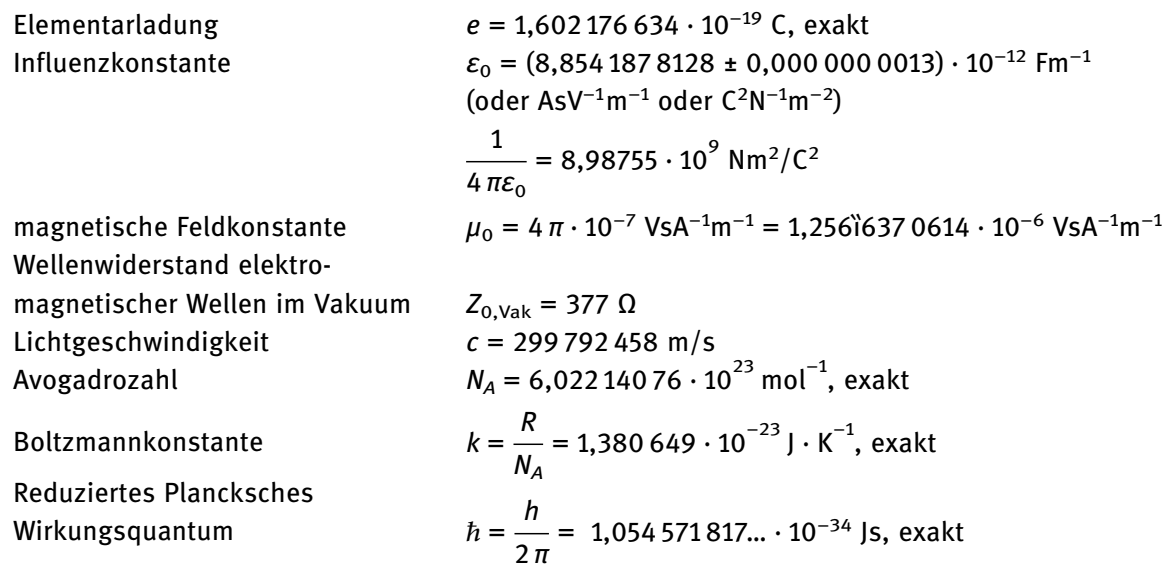


